\title{
Identification of 46 novel SNPs in the 130-kb region containing a myocardial infarction susceptibility gene on chromosomal band 6p21
}

Received: 25 June 2003/ Accepted: 27 June 2003 / Published online: 13 August 2003

(C) The Japan Society of Human Genetics and Springer-Verlag 2003

\begin{abstract}
We identified a total of 187 single-nucleotide polymorphisms (SNPs) at 11 gene loci in the $130-\mathrm{kb}$ region on chromosome $6 \mathrm{p} 21$ containing a gene strongly associated with myocardial infarction (MI). By comparing our data with SNPs deposited in the dbSNP database at the National Center for Biotechnology Information, 46 of these SNPs $(24.6 \%)$ were considered to be novel: four were identified in the P5-1 locus, 14 in the $M I C B$ locus, nine in the BATI locus, one in the $A T P 6 V 1 G 2$ locus, six in the NFKBIL1 locus, one in the $L T A$ locus, one in the TNF locus, five in the $L S T 1$ locus, four in the $L Y 117 a$ locus, and one in the AIF-1 locus. The SNP map presented here should provide as useful resource not only for examining the relationships between genotypes and susceptibility to the MI phenotype, but also for scanning of complex diseases mapped to this local segment on chromosome 6 .
\end{abstract}

Keywords Single-nucleotide polymorphism (SNP) · Fine-scale SNP map - Myocardial infarction critical region $\cdot$ Myocardial infarction susceptibility gene

\section{Introduction}

Despite the changes in life style and development of new pharmacologic approaches, coronary artery dis-

A. Iida $\cdot$ Y. Nakamura

Laboratory for Genotyping, RIKEN SNP Research Center,

Kanagawa, Japan

K. Ozaki $\cdot$ Y. Ohnishi $\cdot$ T. Tanaka

Laboratory for Cardiovascular Diseases,

RIKEN SNP Research Center, Tokyo, Japan

Y. Nakamura $(\square)$

Laboratory of Molecular Medicine, Human Genome Center, Institute of Medical Science, University of Tokyo,

4-6-1 Shirokanedai, Minato-ku, Tokyo 108-8639, Japan

E-mail: yusuke@ims.u-tokyo.ac.jp

Tel.: + 81-35449-5372

Fax: $+81-35449-5433$ eases including myocardial infarction (MI) remain the principal causes of death in the world, indicating the importance of identifying genetic and environmental factors in their pathogenesis (for reviews, see Braunwald 1997; Breslow 1997). By means of a genome-wide gene-based single-nucleotide polymorphism (SNP) analysis and subsequent functional assay, we previously identified the LTA gene as an MI susceptibility gene on $6 \mathrm{p} 21$, and two genetic variants in the gene that may influence lymphotoxin-alpha (LTA) function both quantitatively and qualitatively (Ozaki et al. 2002). During the search for the MI susceptibility gene in this critical region, we attempted to construct a high-density SNP map for linkage disequilibrium (LD) mapping by direct sequencing analysis and identified one extended block of intense LD with LD coefficients drop-off near P5-1 and AIF-1 (Ozaki et al. 2002).

In this paper, we provide a high-resolution SNP map of this MI critical region on $6 \mathrm{p} 21$, in which we have detected a total of 46 novel SNPs among 64 chromosomes from 16 individuals with MI and 16 control individuals.

\section{Subjects and methods}

Blood samples were obtained with written informed consent from 16 individuals with MI and 16 control individuals for this study, which was approved by the ethical committee of the RIKEN SNP Research Center. We generated a reference sequence of $130-\mathrm{kb}$ by assembling sequences Y14768, AP000506, and AC004184 from the GenBank database and then designed primer sets to amplify the 11 gene loci $(A I F-1, L Y 117 a, L S T 1$, $L T B, T N F, L T A, N F K B I L 1, A T P 6 V 1 G 2, B A T 1, M I C B$, and $P 5-1$ ), excluding over most of the regions that corresponded to repetitive sequences predicted by the Repeat Masker program (http://repeatmasker.genome.washington.edu/cgi-bin/RepeatMasker).

Each polymerase chain reaction (PCR) was performed with $20 \mathrm{ng}$ mixed genomic DNA derived from two individuals. Amplification of genomic DNA fragments by PCR, and DNA sequencing of the amplified fragments were performed according to methods described previously (Iida et al. 2003). 


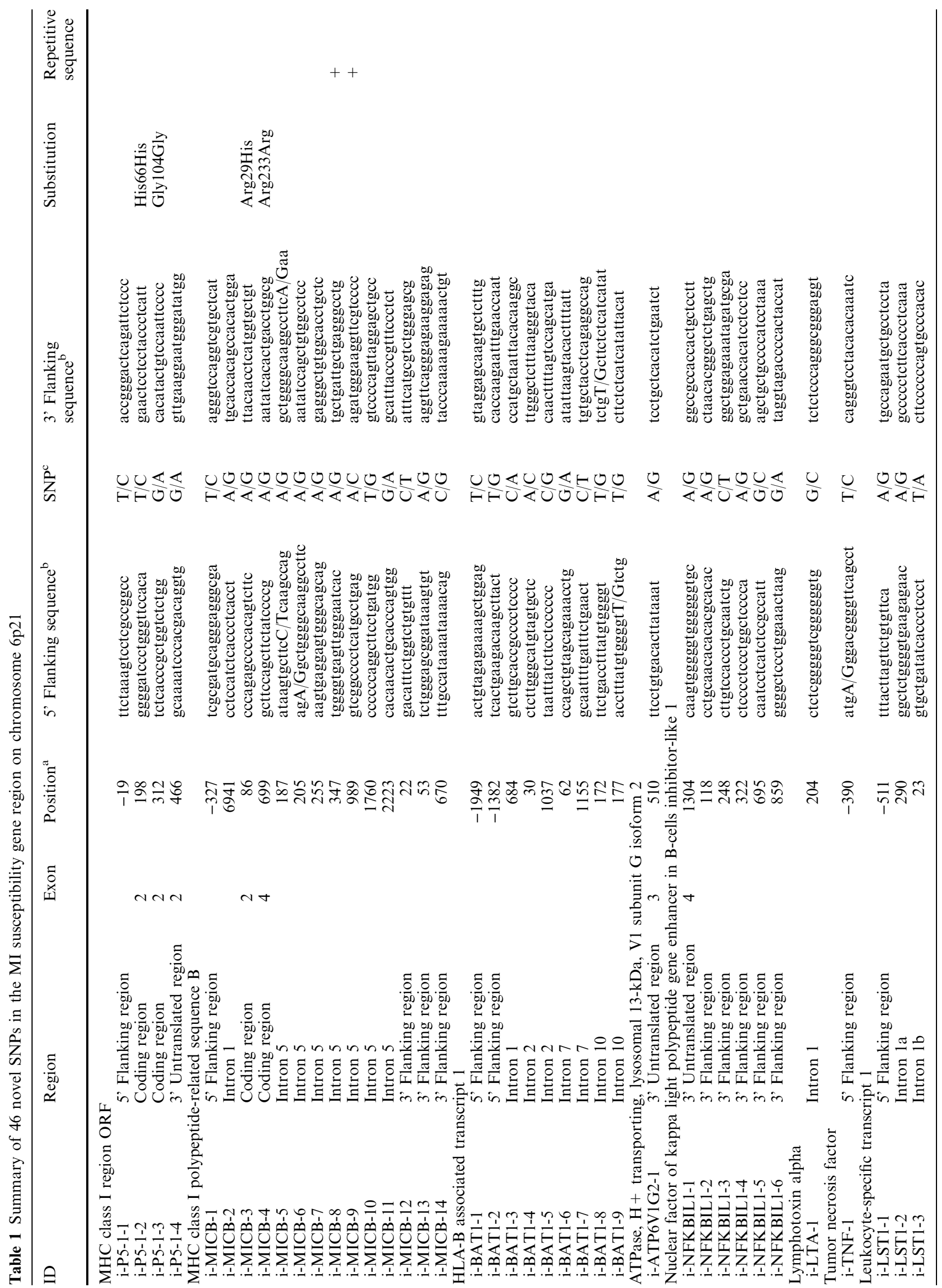



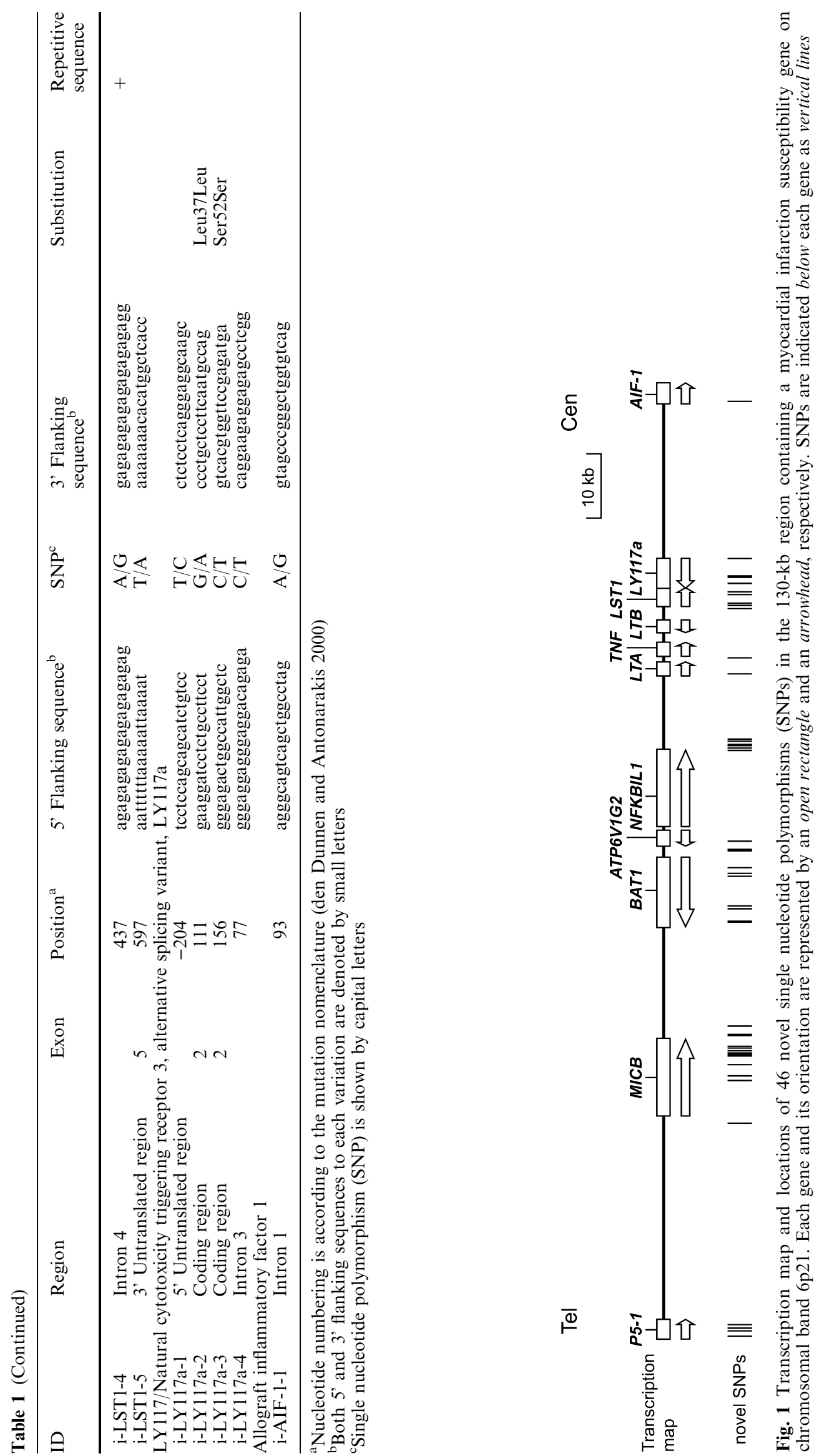


\section{Results and discussion}

By direct sequencing of DNA from 16 individuals with MI and 16 control individuals, we screened SNPs in a 130-kb genomic region corresponding to the MI critical region on $6 \mathrm{p} 21$, which contains 11 genes, except for most of the regions of human repetitive sequences, and identified 187 SNPs. SNPs were distributed every 275 nucleotides on average in the 51.4-kb regions screened, although their densities varied considerably in these regions. We found 68 SNPs in the $M I C B$ gene locus of which $8.6-\mathrm{kb}$ was examined (1 SNP/127 nucleotides), whereas only two were identified in the $3.6-\mathrm{kb}$ genomic region containing the $L T B$ gene (1 SNP/1783 nucleotides). By a comparison of our data with SNPs deposited in the dbSNP database in National Center for Biotechnology Information (NCBI 2003), 46 of the 187 SNPs were considered to be novel (Table 1). Of the 46 SNPs, four were identified in the $P 5-1$ gene locus, 14 in the $M I C B$ gene locus, nine in the $B A T 1$ gene locus, one in the ATP6VIG2 gene locus, six in the NFKBIL1 gene locus, one in the LTA gene locus, one in the $T N F$ gene locus, five in the $L S T 1$ gene locus, four in the LY117a gene locus, and one in the AIF-1 gene locus (Fig. 1, Table 1). The SNPs consisted of 32 transitions and 14 transversions; thus, transitions occurred 2.3 times more frequently than transversions.
The gene-based SNP map presented here should provide as useful resource not only for further examining the relationships between genotypes and susceptibility to the MI phenotype, but also for screening of genes associated with complex diseases mapped to this local segment on chromosome 6 .

\section{References}

Braunwald E (1997) Shattuck lecture - cardiovascular medicine at the turn of the millennium: triumphs, concerns, and opportunities. N Engl J Med 337:1360-1369

Breslow JL (1997) Cardiovascular disease burden increases, NIH funding decreases. Nature Med 3:600-601

Dunnen JT den, Antonarakis SE (2000) Mutation nomenclature extensions and suggestions to describe complex mutations: a discussion. Hum Mutat 15:7-12

Iida A, Saito S, Sekine A, Mishima C, Kitamura Y, Kondo K, Harigae S, Osawa S, Nakamura Y (2003) Catalog of 668 SNPs detected among 31 genes encoding potential drug targets on the cell surface. J Hum Genet 48:23-46

NCBI (2003) dbSNP database (June 2003), http://www.ncbi.nlm. nih.gov/entrez/query.fcgi?db $=$ snp

Ozaki K, Ohnishi Y, Iida A, Sekine A, Yamada R, Tsunoda T, Sato H, Sato H, Hori M, Nakamura Y, Tanaka T (2002) Functional SNPs in the lymphotoxin-alpha gene that are associated with susceptibility to myocardial infarction. Nat Genet 32:650-654 